\title{
Medical abortion for Australian women: it's time
}

\author{
Caroline M De Costa
}

R ecently, a young woman from a rural community presented to a doctor in a small country hospital some distance from her home, requesting termination of pregnancy. She was the mother of two children aged under 3 years, both delivered before 32 weeks gestation because of severe pre-eclampsia. Her partner was unsupportive. At presentation, she was 8 weeks pregnant.

The doctor was sympathetic to her request but was unable to arrange surgical termination in the country hospital. He advised that she would need to travel, by bus and at her own expense, several hundred kilometres to the nearest large town, where an abortion could be performed in a private clinic. The cost would be more than $\$ 700$ in all. This was completely beyond this woman's resources, and she returned home still pregnant.

At 26 weeks of pregnancy, she presented again to the country hospital, severely ill with pre-eclampsia. She was transferred by air to the town in which she might have had the pregnancy terminated, where she underwent emergency caesarean section in a public hospital. The infant died within 24 hours, and the woman spent several days in a highdependency unit. The cost of her transfer and hospitalisation was covered from the public purse.

This woman's story could have been very different if Australian women, like those in the United States, Canada, the United Kingdom, much of Western Europe, Russia, China, Israel, New Zealand, and more recently countries such as Turkey and Tunisia, had access to medical abortion. This woman's story could have been very different if Australian women had access to mifepristone (formerly known as RU-486), a drug which is safe, effective, cheap to produce, and now widely used overseas for medical abortion.

More than 1.5 million early (before 9 weeks) terminations of pregnancy have been performed in Europe, and around 400000 in the US, using mifepristone together with prostaglandin analogues. There is a large body of literature now available on the administration, effectiveness, side effects and risks of the medication, as well as much information about its acceptability to women. ${ }^{1-11}$

\section{Mifepristone and termination of pregnancy}

Mifepristone is a synthetic steroid that blocks the actions of progesterone. It was developed in France in 1980 and underwent clinical trials in France and Switzerland in 1981. ${ }^{1-3,12}$ It was licensed for use in France in 1988, the UK in 1991, and the US in 2000.

The drug can be used for emergency contraception as an alternative to levonorgestrel, as well as to induce menstruation before a period has been missed; it can also be used to bring about abortion, either in early pregnancy or in the second trimester. ${ }^{2,4,12}$ It acts by inhibiting the action of progesterone in the maintenance of early pregnancy, causing degeneration of the decidua, and thus

\footnotetext{
James Cook University School of Medicine, Cairns, QLD.

Caroline M De Costa, FRANZCOG, FRCOG, Professor of Obstetrics and Gynaecology.

Reprints will not be available from the author. Correspondence:

Professor Caroline M De Costa, Department of Obstetrics and

Gynaecology, James Cook University School of Medicine, Cairns

Campus, PO Box 902, Cairns, OLD 4870.
}

\section{ABSTRACT}

- Medical termination of pregnancy with mifepristone, a progesterone antagonist, is available to women in North America, the United Kingdom, much of Western Europe, Russia, China, Israel, New Zealand, Turkey and Tunisia, but not Australia.

- Experience of mifepristone use in around two million abortions has shown that it is safe, effective, cheap to produce, and highly acceptable to women.

- Mifepristone is usually used in combination with a prostaglandin analogue, such as misoprostol; these drugs have been added to the World Health Organization's list of essential medicines for developing countries.

- Availability of this drug in Australia might largely overcome many of the inequities of access to abortion, and is critical for many women in rural areas and women in some ethnic groups whose access to surgical abortion is limited.

MJA 2005; 183: 378-380

the separation of the developing embryo and placenta from the uterine wall; it also causes cervical softening and the release of endogenous prostaglandins. When used for medical termination of pregnancy, mifepristone is usually administered with a prostaglandin analogue (either misoprostol or gemeprost), which brings about expulsion of the uterine contents.

In early pregnancy, various drug regimens have been used, most commonly mifepristone ( $200 \mathrm{mg}$ orally) followed by the prostaglandin analogue misoprostol (one or more doses vaginally or orally), either commencing at the time mifepristone is given or 13 days later. Misoprostol may be given by a clinician or administered by the woman herself. ${ }^{1-3,5-7}$ (Misoprostol is currently available in Australia and, although not listed for gynaecological indications, it is used for cervical dilatation before surgical uterine evacuation and for postpartum haemorrhage; it has also been used overseas to treat incomplete spontaneous abortion.)

In 93\%-98\% of cases, administration of the drug combination leads to complete abortion. In the remainder, the abortion needs to be completed by aspiration of uterine contents by an appropriately qualified doctor. In a very small number of women, heavy vaginal bleeding necessitates hospital admission and sometimes transfusion (transfusion rates of 1 per 1000 and 2.5 per 1000 are cited in various studies). Infection of retained products of conception is a possibility, and antibiotics may be needed. ${ }^{1-3,5-8}$

Mifepristone is also very effective for second trimester termination, again in conjunction with misoprostol or another prostaglandin analogue.

\section{The context of use}

All women requesting termination of pregnancy require complete and accurate information about procedures, and counselling relevant to their particular medical, psychological and social situation - this is true of medical as well as surgical abortion. 
In early pregnancy, confirmation that the pregnancy is intrauterine is essential, and any suggestion of ectopic pregnancy necessitates specialist referral. The drugs for medical abortion must be prescribed by a practitioner with appropriate training; the opportunity may also be taken to carry out cervical screening and screening for sexually transmitted infections. Mifepristone should be administered within a medical context, but misoprostol may be given for the woman to self-administer. There is no need for the woman to remain under medical observation; the abortion process can occur at home, but the woman must have access to appropriate and skilled 24-hour emergency help if needed. - $3,7,8^{\text {Analgesia }}$ must be offered (pain and bleeding are a normal part of the process), and the woman should be supported emotionally as much as possible. Follow-up to confirm that all products of conception have been removed is essential, and the woman should be offered contraception and further counselling if needed.

Degree of satisfaction with the method has been widely investigated, with around 90\% of women being "satisfied" or "very satisfied". In particular, the possibility for privacy and the minimally invasive nature of the treatment have made it acceptable to a large number of women. ${ }^{2,5-8}$

In second-trimester termination, overseas experience with mifepristone used under supervision in hospitals or clinics shows that its use has greatly shortened and simplified the process. ${ }^{2,9}$ For women with second-trimester diagnoses of severe fetal abnormalities and those in whom major medical or psychiatric indications for termination exist (who currently comprise most of the small number of women undergoing late termination in Australia, as elsewhere), the drug combination contributes hugely to ameliorating a very difficult and psychologically traumatic process.

\section{Mifepristone in Australia}

It is clear from this summary that medical abortion could be quickly incorporated into the practice of existing Australian abortion services, and could also easily become part of the practice of those gynaecologists, general practitioners and family planning doctors who wished to provide it. In particular, it could become a real and accessible option for women in rural areas.

Most women undergoing early medical abortion do not require any further surgical assistance or intervention, simply the easy availability of such services if needed. Throughout rural Australia, facilities exist in smaller hospitals for the management of women presenting with spontaneous miscarriage; most of these cases are managed by competent GPs, with only the occasional need for transfer to larger hospitals. The management of incomplete medical abortion is similar, with quite modest requirements for services and equipment. Women from more remote areas might need to stay in the closest small town until the abortion was complete, but would not need to travel long distances to surgical abortion clinics. While not all rural GPs would wish to provide medical abortion, a number would, whereas the provision of surgical abortion, with the need for greater training, and time and financial restraints, will never be an option for small rural centres or hospitals.

It is true that there have been some deaths associated with medical abortions. In the US, since 2000, there has been one death from an undiagnosed ruptured ectopic pregnancy, and another death from myocardial infarction in a woman with known heart disease who may have been more suited to surgical termination. ${ }^{3,8}$ While not diminishing the tragedy of any death, neither of these was directly related to the use of mifepristone. ${ }^{3}$ Also, in the past 5 years, there have been four deaths in the US, all in California, from post-abortion sepsis; in two cases Clostridium sordellii was the causative organism, and in two cases investigation is ongoing. 8,10 C. sordellii, a rare pathogen that produces a powerful toxin, is an occasional commensal in the bowel or vagina. It was reported in the American literature as a cause of death after normal or operative vaginal delivery and gynaecological surgery before the introduction of medical abortion to the US. ${ }^{13,14}$ It could be postulated that the women who developed $C$. sordellii infection after medical termination might equally well have developed the infection had their pregnancies proceeded to term. ${ }^{8}$ These deaths — four in close to 400000 abortions in the US — must be seen in the context of overall maternal mortality figures, currently around 12 per 100000 births in the US., 15 Pregnancy is never without risk for any woman, but both surgical and medical abortion carry less than $10 \%$ of the risk of mortality when compared with continuing the pregnancy. ${ }^{15}$

In 1999, the International Federation of Obstetrics and Gynaecology (FIGO) stated that "after appropriate counselling, a woman has the right to have access to medical or surgical induced abortion, and ... healthcare services have an obligation to provide such services as safely as possible." 2 This view was supported by the World Health Organization which, in 2003, published Safe abortion: technical and policy guidance to assist health care providers to make surgical and medical abortion safe and accessible. ${ }^{9}$ More recently, WHO has added mifepristone and misoprostol to its list of essential medicines for developing countries, medicines it believes "satisfy the priority healthcare needs of the population". ${ }^{16}$ Both the UK Royal College of Obstetricians and Gynaecologists and the American College of Obstetricians and Gynecologists have been outspoken in their support for the introduction of medical abortion in their respective domains, seeing it as an important health care need for women. ${ }^{1-3,8}$ In Australia to date, there have been efforts by individual women and health care professionals, but support for medical abortion by relevant professional bodies has been muted.

In an article in the Journal in 2004, de Crespigny and Savulescu examined the discrepancies in abortion laws between Australian states, and argued the case for uniform decriminalisation of existing laws. ${ }^{17}$ In addition, they pointed out that, although abortion is legal in some situations in all states, there are marked inequities of access to existing services. These inequities might be largely overcome by the introduction of medical abortion in this country.

The topic of abortion is an emotive and controversial one. Recent media debate seems to have died down, and no doubt politicians simply hope the issue will go away. It will not. We do not know how many abortions are performed in Australia each year, but it is believed the number is large ${ }^{18}$ Certainly, it would be desirable to try to reduce the figure, whatever it is, by appropriate research and better provision of contraception. However, given the complex and compelling nature of human sexuality, unwanted pregnancies will continue to occur, and Australian women will continue to seek safe, legal abortion. It is worth noting that, in European countries where mifepristone has been available for some time, there has been no increase in the number or rate of abortions overall, but the proportion of abortions performed at earlier gestations has risen. The safety of abortion has been shown to be directly related to how early in pregnancy it is performed. ${ }^{15}$ 
The case for medical abortion in Australia should be judged not on political grounds but solely on evidence-based medical criteria. As de Crespigny and Savulescu state, there appears to be broad community support for a woman's right to choose an abortion after appropriate discussion with her doctor. For many women in urban areas, the ability to choose medical rather than surgical abortion would simply bring their choices into line with current practice in most of the rest of the developed world. However, for rural women and for women of some ethnic groups for whom privacy is a particular issue, the option of medical abortion is critical if they are to be treated equally with other Australian women.

Recently, we have seen the rapid introduction of sildenafil and other similar drugs into the Australian pharmacopoeia. We live in a society where many aspects of sexual behaviour are freely discussed and promoted. This includes the treatment of erectile dysfunction and the recognition of the importance of this subject to many men whose sexual lives have been enhanced by the availability of these agents. At the same time, although unplanned and unwanted pregnancies are a direct consequence of sexual activity, we are as a society much less willing to acknowledge that provision of safe, accessible abortion facilities is essential to allow women control of their reproductive lives. It's time to face these facts, and to add mifepristone to the range of choices that can be offered to Australian women.

\section{Competing interests}

None identified.

\section{References}

1 American College of Obstetricians and Gynecologists. Recommendations on medical abortions issued to US obstetricians-gynecologists, 31 March 2001 [news release]. Available at: http://www.acog.org/from_ home/publications/press_releases/nr03-31-01-2.cfm (accessed Jun 2005).

2 Royal College of Obstetricians and Gynaecologists. National clinical guidelines. The care of women requesting induced abortion. London: RCOG, 2004

3 Dickerson V, American College of Obstetricians and Gynecologists. ACOG letter on safety of RU-486, 7 December 2004 [news release].
Available at: http://www.acog.org/from_home/publications/press_ releases/nr12-07-04.cfm (accessed Jun 2005).

4 Sparrow MJ. A woman's choice. Aust N Z J Obstet Gynaecol 2004; 44: 88 92.

5 Shannon CS, Winikoff B, Hausknecht R, et al. Multicenter trial of a simplified mifepristone medical abortion regimen. Obstet Gynecol 2005; 105: 345-351.

6 Schreiber CA, Creinin MD, Harwood B, Murthy AS. A pilot study of mifepristone and misoprostol administered at the same time for abortion in women with gestation from 50 to 63 days. Contraception 2005; 71: 447450.

7 Faucher B, Baunot N, Madelenat P. The efficacy and acceptability of mifepristone medical abortion with home administration misoprostol provided by private providers linked to the hospital: a prospective study of 433 patients. Gynecol Obstet Fertil 2005; 33: 220-227.

8 Hausknecht R. Mifeprex and misoprostol for early abortion: 41/2 years experience in the US. Presentation at the Annual Clinical Meeting of American College of Obstetricians and Gynecologists; 9 May 2005; San Francisco, Cal.

9 World Health Organization. Safe abortion: technical and policy guidance. Geneva: WHO, 2003

10 Centers for Disease Control and Prevention (CDC). Clostridium sordellii toxic shock syndrome after medical abortion with mifepristone and intravaginal misoprostol - United States and Canada, 2001-2005. MMWR Morb Mortal Wkly Rep 2005; 54: 724.

11 Ashok PW, Flett GM, Templeton A. Termination of pregnancy at 9-13 weeks' amenorrhoea with mifepristone and misoprostol. Lancet 1998; 352: 542-543.

12 Baulieu EE. Contraception by the progesterone antagonist RU 486: a novel approach to human fertility control. Res Reprod 1987; 19: 3-4.

13 Hogan SF, Ireland K. Fatal acute spontaneous endometritis resulting from Clostridium sordelli. Am J Clin Pathol 1989; 91: 104-106.

14 McGregor JA, Soper DE, Lovell G, Todd JK. Maternal deaths associated with Clostridium sordellii infection. Am J Obstet Gynecol 1989; 161: 987995.

15 Bartlett L, Berg C, Shulman H, et al. Risk factors for legal induced abortion-related mortality in the United States. Obstet Gynecol 2004; 103: 729-737.

16 Gibson L. WHO puts abortifacients on its essential drugs list. BMJ 2005; 331: 68.

17 De Crespigny LJ, Savulescu J. Abortion: time to clarify Australia's confusing laws. Med J Aust 2004; 181: 201-203.

18 Chan A, Sage LC. Estimating Australia's abortion rates 1985-2003. Med J Aust 2005; 182: 447-452.

(Received 1 Jun 2005, accepted 16 Aug 2005)

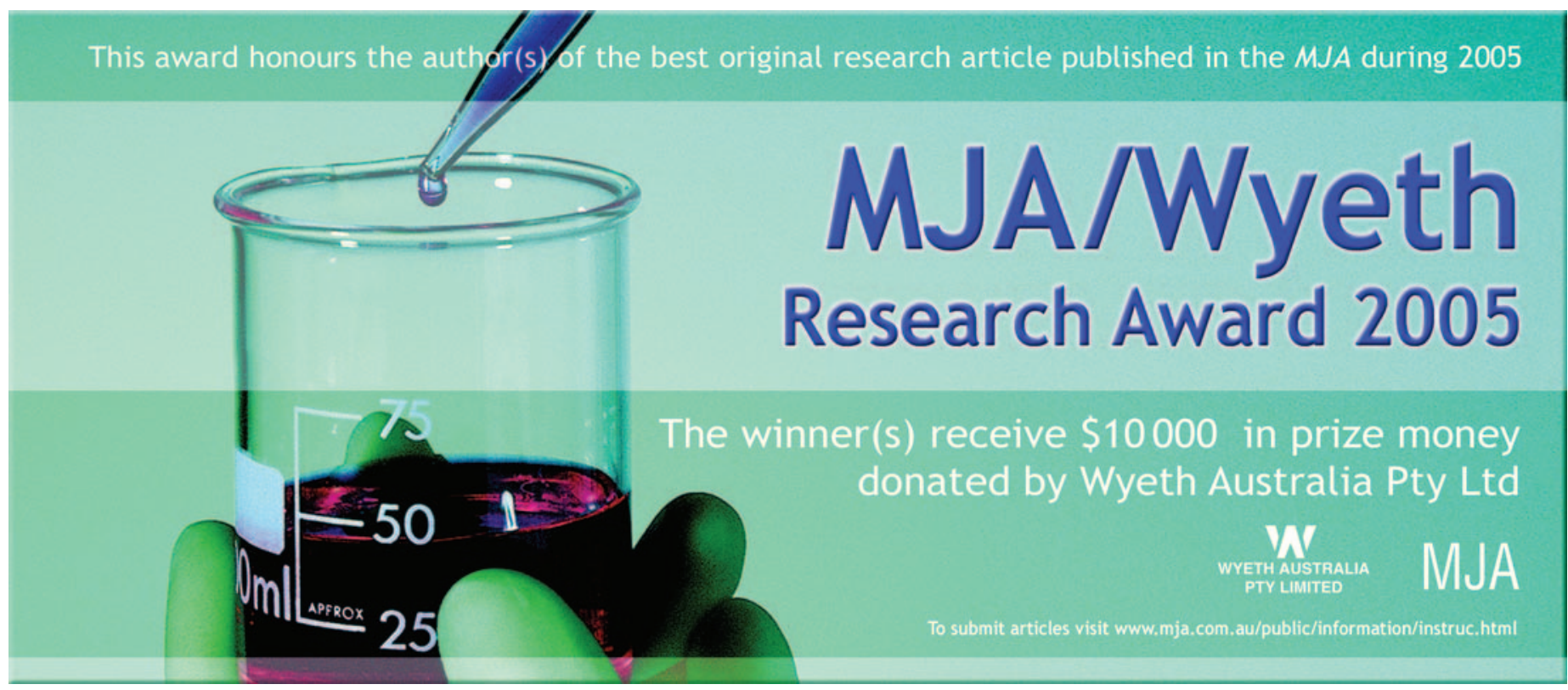


ADVERT

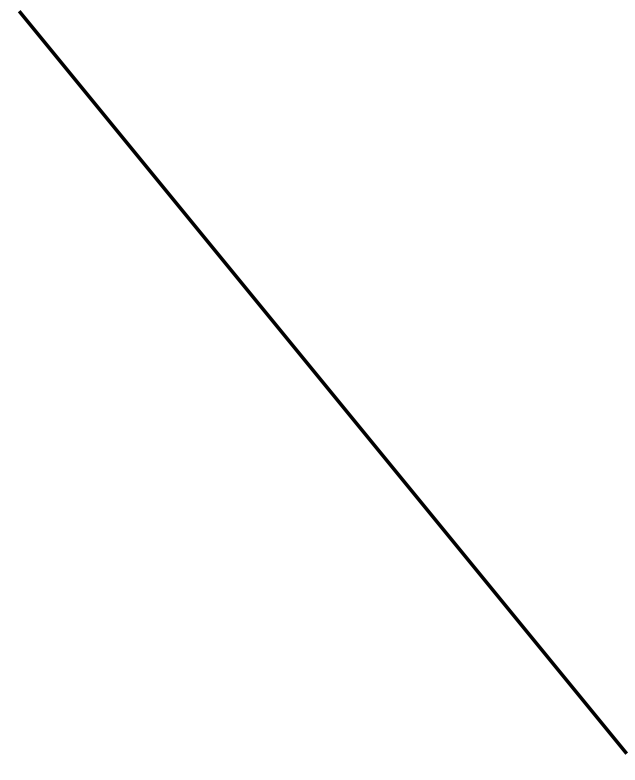

Lipidil

page 381 